\title{
Design of a distributed finite-time observer using observability decompositions
}

\author{
Haik Silm ${ }^{1,2,3}$, Rosane Ushirobira ${ }^{2}$, Denis Efimov ${ }^{2,4}$, Wim Michiels ${ }^{3}$ and Jean-Pierre Richard ${ }^{1,2}$
}

\begin{abstract}
In this paper, a distributed observer is presented to estimate the state of a linear time-invariant plant in finite-time in each observer node. The design is based on a decomposition into locally observable and unobservable substates and on properties of homogeneous systems. Each observer node can reconstruct in finite-time its locally observable substate with its measurements only. Then exploiting the coupling, a finite-time converging observer is constructed for the remaining states by adding the consensus terms. A numerical example illustrates the result.
\end{abstract}

\section{INTRODUCTION}

Driven by the fact that numerous collaborative approaches are proposed to reach efficiency in complex systems like power networks, traffic systems and robot swarms etc., the research on distributed observers has gained more attention in recent years. The idea is to take advantage of the fact that smart multi-agent systems are deployed almost everywhere with sensing, processing and communication capabilities. This leads to new opportunities for the task of state estimation but also to related fields as synchronization, which is a fundamental problem in interconnected systems, and can be represented as the problem of designing observers [1].

In this context, distributed state estimation [2] has been proposed as an elementary problem. In this framework a general linear plant with multiple measurement outputs is observed by a sensor network. Each sensor acts as an observer node, with a complete model of the plant, and the aim is to reconstruct the state of the plant in each node. Since the system is not observable from a single output, the observer nodes exchange information with each other, subject to a communication graph, to obtain the estimate of the full plant state, i.e. to achieve state-omniscience [3]), without falling back to a central fusion framework (as it has been shown in [4] the distributed solution outperforms the centralized one when taking into account communication lags).

In the present note, to tackle this problem we will rely on the observability decomposition of the state space. This is inspired by the fact that each observer node has some part of the state space which is observable by its measurement, and that an independent observer can be designed (for the locally observable substate). This has been first exploited in [5] and also by other authors, for example in [6] to achieve a guaranteed exponential convergence rate of the observer. Recently, in [7] a design based on a multi-hop subspace

${ }^{1}$ Centrale Lille, 59000 Lille, France

2 Inria, Univ. Lille, CNRS, UMR 9189 - CRIStAL, 59000 Lille, France

${ }^{3}$ Department of Computer Science, KU Leuven, 3001 Heverlee, Belgium

4 Department of Control Systems and Informatics, University ITMO,

197101 Saint Petersburg, Russia decomposition is presented which makes it possible to assign the poles of the error system at will. A related approach is suggested in [8], but with a staircase decomposition for multi-sensors.

For the remaining part, we assume that there exists a proper communication graph. A widely adopted approach is to use a consensus mechanism with neighboring agents. Since initially proposed for Distributed Kalman Filtering [9] many methods have been presented to design the consensus gains, for example in [10] using $\mathcal{H}_{\infty}$ filter technique.

In contrast to the above works which try to achieve a desired asymptotic convergence rate an alternative approach is to design distributed observers with respect to the finite-time stability property [11]. Centralized finite-time observers have been suggested before in [12]-[14] based on homogeneity, which have the advantages that compared to asymptotically converging observers robustness in terms of disturbances is retained while next to finite-time stability, robustness to timedelays is achieved [15], which is important in the context of networked observers. Additionally, in a mobile environment the communication topology can be changed at some timeinstants, making it more important that time-constraints are met. Time-varying communication graphs and finite-time convergence have also been examined in [16], however for a discrete-time system akin to a dead-beat observer.

In this work, following [11], we propose a distributed finite-time observer for continuous-time systems, but to include general linear plant dynamics, we additionally exploit the decoupling property of the observability decomposition due to finite-time convergences of some part of the system.

The outline of the paper is as follows. In the preliminaries the finite-time observer design is presented for the case of a centralized observer and the observability decomposition is recapitulated. These results are used in the main part to introduce the distributed finite-time observer design for the observable part and later for the unobservable part. For the unobservable part first an exponentially converging observer with a guaranteed convergence rate is presented, then under an assumption the observer is designed to be finite-time converging with favorable robustness properties. An example in the last section illustrates the result.

Notation:

- For $x=\left[\begin{array}{lll}x_{1} & \ldots & x_{n}\end{array}\right]^{\mathrm{T}} \in \mathbb{R}^{n}$, we denote by $\operatorname{diag}(x) \in \mathbb{R}^{n \times n}$ the diagonal matrix with $x_{1}, \ldots, x_{n}$ on the main diagonal and 0 otherwise. Also we denote by $\lceil x\rfloor^{\alpha}=\left[\begin{array}{lll}\operatorname{sign}\left(x_{1}\right)\left|x_{1}\right|^{\alpha_{1}} & \ldots & \operatorname{sign}\left(x_{n}\right)\left|x_{n}\right|^{\alpha_{n}}\end{array}\right]^{\mathrm{T}}$ the sign preserving element-wise exponentiation, with $\alpha=\left[\begin{array}{lll}\alpha_{1} & \ldots & \alpha_{n}\end{array}\right]^{\mathrm{T}} \in \mathbb{R}_{+}^{n}$.

- $1_{n} \in \mathbb{R}^{n}$ is the vector with all entries equal to 1 . 
- $E_{n} \in \mathbb{R}^{1 \times n}$ is the unit row vector $E_{n}=\left[\begin{array}{lll}1 & \ldots & 0\end{array}\right]$.

- For all $n \geq 2$, denote $\mathcal{J}_{n}=\left[\begin{array}{cccc}0 & 1 & \ldots & 0 \\ & \ddots & \ddots & \vdots \\ & & \ddots & 1 \\ 0 & & \ldots & 0\end{array}\right]$.

- A block column matrix formed by matrices $C_{1}, \ldots, C_{N} \in \mathbb{R}^{1 \times n}$ is denoted by $\operatorname{col}\{C\} \in \mathbb{R}^{N \times n}$.

- A block diagonal matrix formed by matrices $P_{1}, \ldots, P_{N} \in \mathbb{R}^{n \times n}$ is denoted by $\bar{P} \in \mathbb{R}^{N n \times N n}$.

- $\mathcal{N}_{i}$ denotes the set of neighboring nodes of node $i$

\section{Preliminaries}

\section{A. Finite-Time observers}

First we revise the design of a centralized finite-time observer for a linear time-invariant system

$$
\dot{x}=A x, \quad y=C x,
$$

with state $x \in \mathbb{R}^{n}$, dynamics matrix $A \in \mathbb{R}^{n \times n}$ and output matrix $C \in \mathbb{R}^{1 \times n}$.

It is well-known that if the rank of the observability matrix $\mathcal{O}(A, C)$ is $n$, then the system is observable and using a Luenberger observer, its state can be estimated with arbitrary asymptotic convergence rate. Additionally, there exists a state transformation $x=T_{c} v, T_{c} \in \mathbb{R}^{n \times n}$ which describes the system as an integrator chain plus additional output feedback (the observer canonical form [17])

$$
\dot{v}=T_{c}{ }^{-1} A T_{c} v=\mathcal{J}_{n} v-a y,
$$

with $y=C T_{c} v=v_{1}$ and where $a \in \mathbb{R}^{n}$ consists of the coefficients of the characteristic polynomial of $A$.

Following the works of [18] and [14], there exists a nonlinear observer for the system (1) of the form

$$
\dot{\tilde{x}}=\tilde{A} \tilde{x}-\tilde{a} y-\widetilde{L}\left\lceil 1_{n}(C \tilde{x}-y)\right\rfloor^{\gamma}
$$

with $\widetilde{A}=T_{c} \mathcal{J}_{n} T_{c}^{-1}, \tilde{a}=T_{c} a, \widetilde{L}=T_{c} \operatorname{diag}(L)$ and entries of $\gamma \in \mathbb{R}^{n}$ as $\gamma_{s}=1+s \nu, s=1, \ldots, n$ where the gains $L \in \mathbb{R}^{n}$ and $1 / n<\nu<0$ can be designed in a way that the estimate $\tilde{x}$ converges to $x$ in a finite time.

Theorem 1 ([11]]): Consider the system (1) with $\operatorname{rank} \mathcal{O}(A, C)=n$ and the observer (3). If

1) there exists $L \in \mathbb{R}^{n}$ and positive definite matrices $Q, P \in \mathbb{R}^{n \times n}$ satisfying the Lyapunov equation

$$
\left(\mathcal{J}_{n}-L E_{n}\right)^{\mathrm{T}} P+P\left(\mathcal{J}_{n}-L E_{n}\right)+Q=0,
$$

$$
\begin{aligned}
& \text { 2) }-\frac{\eta}{n(\sqrt{n}+\eta)}<\nu<0 \text { with } \eta= \\
& \frac{\exp (1)}{2} \frac{\lambda_{\min }(Q)}{\lambda_{\max }(P)\|P \operatorname{diag}(L)\|},
\end{aligned}
$$

where $\lambda_{\min }(Q), \lambda_{\max }(P)$ are the smallest and largest eigenvalue of $Q$ and $P$, respectively, then the dynamics of the error $e=\tilde{x}-x$ is globally finite-time stable.

It is worth stressing that despite the estimation problem (1) has been posed without state disturbances, measurement noises and delays, due to homogeneity of the estimation error dynamics, the proposed observer (3) is ubiquitously robust with respect to all these perturbations [15], [19].

\section{B. Observability decomposition and problem statement}

Consider a system with $N>1$ outputs

$$
\dot{x}=A x+d_{1}, \quad y_{i}=C_{i} x+d_{2}, \quad i=1, \ldots, N
$$

with dynamics matrix $A \in \mathbb{R}^{n \times n}$ and output matrices $C_{i} \in$ $\mathbb{R}^{1 \times n}$ and where $d=\left[\begin{array}{llll}d_{1}^{\mathrm{T}} & d_{2,1}^{\mathrm{T}} & \ldots & d_{2, N}^{\mathrm{T}},\end{array}\right]^{\mathrm{T}}$ is the vector representing the external disturbance and measurement noises, which are assumed to be essentially bounded functions of time. For each output $y_{i}$, the observable subspace has dimension $r_{i}=\operatorname{rank} \mathcal{O}\left(A, C_{i}\right)$ and the unobservable subspace may be unstable, hence the system is not detectable from any single output $y_{i}$.

We decompose now the system into observable and unobservable substates. Choosing a coordinate transformation $x=T_{i}\left[\begin{array}{c}x_{o, i} \\ x_{u, i}\end{array}\right]$, where $T_{i}=\left[T_{o, i} T_{u, i}\right]$ and $T_{u, i}$ is a submatrix whose columns form a basis of $\operatorname{ker} \mathcal{O}\left(A, C_{i}\right) \in \mathbb{R}^{n \times\left(n-r_{i}\right)}$, gives

$$
T_{i}^{\mathrm{T}} A T_{i}=\left[\begin{array}{cc}
A_{o, i} & 0 \\
A_{r, i} & A_{u, i}
\end{array}\right], \quad C_{i} T_{i}=\left[\begin{array}{ll}
C_{o, i} & 0
\end{array}\right] .
$$

In the new representation, the observable subsystem

$$
\dot{x}_{o, i}=A_{o, i} x_{o, i}+d_{o, i}, \quad y_{i}=C_{o, i} x_{o, i}+d_{2, i}
$$

is independent from $x_{u, i}$, while the unobservable subsystem

$$
\dot{x}_{u, i}=A_{r, i} x_{o, i}+A_{u, i} x_{u, i}+d_{u, i}
$$

does not affect the output $y_{i}$; here $d_{o, i}$ and $d_{u, i}$ are transformed disturbances $d_{1}=T_{i}\left[\begin{array}{l}d_{o, i} \\ d_{u, i}\end{array}\right]$. The goal of the distributed observer design is to have estimates $\tilde{x}_{o, i}$ and $\tilde{x}_{u, i}$ which converge towards the real state of the plant with non-asymptotic rates in the disturbance-free case, and have bounded errors (e.g. in the input-to-state stability (ISS) sense) in the presence of $d$. To this end we will use the measurement output for the observable subspaces, while for the unobservable subspaces we will have to rely on information exchange between the nodes.

\section{Distributed ObSeRver}

By design, an observable subsystem (6) can be transformed with a transformation matrix $T_{c, i} \in \mathbb{R}^{r_{i} \times r_{i}}$ into observer canonical form and consequently as in Theorem 1 the observable substate $x_{o, i}$ can be estimated in finite-time with the observer

$$
\dot{\tilde{x}}_{o, i}=\widetilde{A}_{o, i} \tilde{x}_{o, i}-\tilde{a}_{o, i} y_{i}-\widetilde{L}_{o, i}\left\lceil 1_{n}\left(C_{o, i} \tilde{x}_{o, i, 1}-y_{i}\right)\right]^{\gamma_{o, i}},
$$

where $\widetilde{A}_{o, i}=T_{c, i} \mathcal{J}_{n} T_{c, i}^{-1}, \quad \tilde{a}_{i}=T_{c, i} a_{i}, \quad \widetilde{L}_{o, i}=$ $T_{c, i} \operatorname{diag}\left(L_{o, i}\right), a_{i} \in \mathbb{R}^{r_{i}}$ consists of the coefficients of the characteristic polynomial of $A_{o, i}, \gamma_{o, i} \in \mathbb{R}^{r_{i}}, \gamma_{o, i, s}=$ $1+s \nu_{o, i}, s=1, \ldots, r_{i}$, and tuning parameters $L_{o, i} \in \mathbb{R}^{r_{i}}$, $\nu_{o, i} \in\left(-r_{i}^{-1}, 0\right)$.

Corollary 2: Consider the system (4) with state transformations for the observability decomposition (5) and observers (8) for the observable substate of each observer node, $i=1, \ldots, N$. If 
1) $L_{o, i}$ is such that there exists positive definite matrices $Q_{i}, P_{i} \in \mathbb{R}^{r_{i} \times r_{i}}$ satisfying the Lyapunov equation

$2)-\frac{\eta_{i}}{r_{i}\left(\sqrt{r_{i}}+\eta_{i}\right)}<\nu_{o, i}<0$
$\frac{\exp (1)}{2} \frac{\lambda_{\min }\left(Q_{i}\right)}{\lambda_{\max }\left(P_{i}\right)\left\|P_{i} \operatorname{diag}\left(L_{o, i}\right)\right\|}$ then the dynamics of the errors $e_{o, i}=\tilde{x}_{o, i}-x_{o, i}$ are globally finite-time stable for the case $d=0$ and finite-time ISS with respect to the input $d$ otherwise.

Proof: The claim about finite-time stability is a direct consequence of Theorem 1. The finite-time ISS property with respect to $d$ follows from homogeneity of the estimation error dynamics with negative degree [19].

\section{A. Asymptotic observer for the unobservable subspace}

For simplicity it is assumed that $d=0$ and that there is no delay in the communication of the nodes (they will be included in the next subsection). With $\left[\begin{array}{l}x_{o, j} \\ x_{u, j}\end{array}\right]$ as the state in the observability decomposed form of some node $j$, there always exist $F_{i, j}$ and $G_{i, j}$ for which

$$
x_{u, i}=F_{i, j} x_{o, j}+G_{i, j} x_{u, j} .
$$

Then the linear observers

$$
\dot{\tilde{x}}_{u, i}=A_{r, i} \tilde{x}_{o, i}+A_{u, i} \tilde{x}_{u, i}-K_{i} \Phi_{i}
$$

with the consensus term

$$
\Phi_{i}=\sum_{j \in \mathcal{N}_{i}} \tilde{x}_{u, i}-F_{i, j} \tilde{x}_{o, j}-G_{i, j} \tilde{x}_{u, j},
$$

will lead to exponential convergence of the estimation error $e_{u, i}=\tilde{x}_{u, i}-x_{u, i}$ with convergence rate $\alpha$ in the perturbation free case if $K_{i}$ are selected as follows:

Theorem 3: Consider the system (4) with detectable pair $\left(A, \operatorname{col}\left\{C_{i}\right\}_{i=1}^{N}\right)$ and state-transformation (5). Assume that there exist matrices $P_{i}>0, Y_{i}, i=1, \ldots, N$ for which the LMI $\Psi \leq 0$ with diagonal blocks

$$
\Psi_{i i}=P_{i} A_{u, i}+A_{u, i}^{\mathrm{T}} P_{i}+p_{i} Y_{i}+p_{i} Y_{i}^{\mathrm{T}}+\alpha P_{i}
$$

and off-diagonal blocks

$$
\Psi_{i j}=\Psi_{i j}^{\mathrm{T}}=Y_{i}+Y_{j}^{\mathrm{T}}, Y_{i}=0 \text { if } j \notin \mathcal{N}_{i}
$$

is satisfied for $\alpha>0$. Then the distributed observers (10) with 11] can be designed for the unobservable subspaces with $K_{i}=\left(Y_{i}^{\mathrm{T}}\right)^{-1} P_{i}$ such that the dynamics of the errors $e_{u, i}=\tilde{x}_{u, i}-x_{u, i}$ are globally exponentially stable with rate $\alpha$ if $e_{o, i} \rightarrow 0$ and $\bar{d}_{i}=0, \tau_{i, j}=0$ for all $j \in \mathcal{N}_{i}$ and $i=1, \ldots, N$.

Proof: If $e_{o, i}$ is zero, then the dynamics for the unobservable substate is

$$
\dot{e}_{u, i}=A_{u, i} e_{u, i}-p_{i} K_{i} e_{u, i}+K_{i} \sum_{j \in \mathcal{N}_{i}} G_{i, j} e_{u, j}
$$

Let $V=E_{u, i}^{\mathrm{T}} \bar{P} E_{u, i}, E_{u, i}^{\mathrm{T}}=\left[\begin{array}{lll}e_{u, 1} & \ldots & e_{u, N}\end{array}\right]^{\mathrm{T}}$ be a Lyapunov-function, then its derivative is

$$
\dot{V}=E_{u, i}^{\mathrm{T}}\left(\bar{P}\left(\overline{A_{u}}-\mathcal{K}\right)+\left(\overline{A_{u}}-\mathcal{K}\right)^{\mathrm{T}} \bar{P}\right) E_{u, i},
$$

where $\mathcal{K}=$

$$
\left[\begin{array}{cccc}
p_{i} K_{1} & -K_{1} G_{1,2} & \cdots & -K_{1} G_{1, N} \\
& & \ddots & \\
-K_{N} G_{N, 1} & \ldots & -K_{N} G_{N, N-1} & p_{i} K_{N}
\end{array}\right],
$$

with the off-diagonal blocks $\mathcal{K}_{i j}=0$ if $j \notin \mathcal{N}_{i}$. With the substitution $Y_{i}=P_{i} K_{i}$ the inequality $\dot{V}+\alpha V \leq 0$ leads to the above LMI. For non-zero but vanishing $e_{o, i}$ the global asymptotic stability is then retained due to ISS arguments for the linear dynamics 12 .

In the case of perturbations the standard linear system results apply: boundedness of the estimation errors for bounded $\bar{d}_{i}(t)$, asymptotic stability for small delays $\tau_{i, j}$, otherwise instability.

\section{B. Finite-time observer for the unobservable subspace}

After presenting an observer with exponential convergence of the unobservable part, in this section we will design a complete finite-time distributed observer under the following assumption:

Assumption 1: For each observer node, the unobservable substates can be expressed as

$$
x_{u, i}=\sum_{j \in \mathcal{N}_{i}} F_{i, j} x_{o, j}
$$

for some $F_{i, j} \in \mathbb{R}^{\left(n-r_{i}\right) \times r_{j}}$.

For a complete communication graph this is always true if the observability matrix of the pair $\left(A, \operatorname{col}\left\{C_{i}\right\}_{i=1}^{N}\right)$ has full rank. The validity in other cases depends on the graph topology and the output matrices for each node.

Let $\tilde{x}_{u, i}$ denote the estimate for the unobservable substate $x_{u, i}$. According to Corollary 1 , the estimates $\tilde{x}_{o, i}$ converge to $x_{o, i}$ subject to a bounded error originated by the presence of $d$, then from Assumption 1 a direct way to obtain the remaining substate is by

$$
\tilde{x}_{u, i}(t)=\sum_{j \in \mathcal{N}_{i}} F_{i, j} \tilde{x}_{o, j}\left(t-\tau_{i, j}\right)+\bar{d}_{i}(t),
$$

where $\tau_{i, j} \geq 0$ and $\bar{d}_{i}$ denote communication delays and unknown noise, respectively.

The noise $\bar{d}_{i}$ represents a cumulative effect of $d$ through the corresponding observable variables on the estimate $\tilde{x}_{u, i}$; it is absent in the disturbance-free case (i.e. when $d=$ 0 ) and in the presence of $d$ it is an essentially bounded function of time, with the maximal amplitude related with the corresponding value of $d_{o, i}$ and $d_{2, i}$ in (6). The delays $\tau_{i, j}$ are originated by the distributed nature of (6) and networked communications between the nodes. For the observable part, it has been assumed that the observer is implemented locally at the sensor level, and a possible delay may be neglected since there is no long-distance communication (which is a reasonable assumption we make for simplicity, but due to the aforementioned robustness it is actually not necessary).

Therefore, for communicating nodes in the context of distributed observers for large-scale systems, these delays and noises are technically inevitable, which means that the 
error $e_{u, i}(t)=\tilde{x}_{u, i}(t)-x_{u, i}(t)=\sum_{j \in \mathcal{N}_{i}} F_{i, j}\left(\tilde{x}_{o, j}(t-\right.$ $\left.\left.\tau_{i, j}\right)-x_{o, j}(t)\right)+\bar{d}_{i}(t)$ will never be zero in their presence. Consequently, since $x_{u, i}(t)$ is a dynamic variable, with a known (up to disturbance $d_{u, i}$ ) dynamics, it is a common approach in such a case to fuse the indirect measurements from other nodes using a filter [20], [21], which in our case will be designed first as a finite-time observer.

Introducing the consensus term

$$
\Psi_{i}(t)=\sum_{j \in \mathcal{N}_{i}} \frac{1}{p_{i}} \tilde{x}_{u, i}(t)-F_{i, j} \tilde{x}_{o, j}\left(t-\tau_{i, j}\right)-\bar{d}_{i}(t),
$$

where $p_{i}$ is the cardinality of $\mathcal{N}_{i}$, the observer is chosen as

$$
\begin{aligned}
\dot{\tilde{x}}_{u, i}(t)=A_{r, i} \tilde{x}_{o, i}(t) & +A_{u, i} \tilde{x}_{u, i}(t)+K_{i} \Psi_{i}(t) \\
& -\operatorname{diag}\left(L_{u, i}\right)\left\lceil 1_{n-r_{i}} \Psi_{i, 1}(t)\right\rfloor^{\gamma_{u, i},}
\end{aligned}
$$

with $K_{i} \in \mathbb{R}^{\left(n-r_{i}\right) \times\left(n-r_{i}\right)}, L_{u, i} \in \mathbb{R}^{n-r_{i}}, \gamma_{u, i} \in \mathbb{R}^{n-r_{i}}$, $\gamma_{u, i, s}=1+s \nu_{u, i}, s=1, \ldots, n-r_{i}$, and $\nu_{u, i} \in\left(-\frac{1}{n-r_{i}}, 0\right)$ be selected as follows:

Corollary 4: Consider the system (4) with state transformations for the observability decomposition (5) and observers (16) with 15) for the unobservable substate of each observer node. Let Assumption 1 be satisfied, $\tilde{x}_{o, i}(t)=$ $x_{o, i}(t)$ for all $t \geq 0$ and $\bar{d}_{i}=0, \tau_{i, j}=0$ for all $j \in \mathcal{N}_{i}$ and $i=1, \ldots, N$. If

1) $K_{i}=\mathcal{J}_{n-r_{i}}-A_{u, i}$

2) $L_{u, i}$ are selected such there exists positive matrices $Q_{i}, P_{i} \in \mathbb{R}^{\left(n-r_{i}\right) \times\left(n-r_{i}\right)}$ satisfying the Lyapunov equation

$$
\left(\mathcal{J}_{\left(n-r_{i}\right)}-L_{u, i} E_{n-r_{i}}\right)^{\mathrm{T}} P_{i}+P_{i}\left(\mathcal{J}_{r_{i}}-L_{u, i} E_{n-r_{i}}\right)+Q_{i}=0,
$$

3) $-\frac{\eta_{i}}{\left(n-r_{i}\right)\left(\sqrt{\left(n-r_{i}\right)}+\eta_{i}\right)}<\nu_{u, i}<0$ with $\eta_{i}=$ $\frac{\exp (1)}{2} \frac{\lambda_{\min }\left(Q_{i}\right)}{\lambda_{\max }\left(P_{i}\right)\left\|P_{i} \operatorname{diag}\left(L_{u, i}\right)\right\|}$,

then the dynamics of the errors $e_{u, i}=\tilde{x}_{u, i}-x_{u, i}$ are globally finite-time stable.

Proof: With (7) and (16) the error dynamics of the unobservable parts are

$\dot{e}_{u, i}=A_{r, i} e_{o, i}+A_{u, i} e_{u, i}+K_{i} \Psi_{i}-\operatorname{diag}\left(L_{u, i}\right)\left\lceil 1_{n-r_{i}} \Psi_{i, 1}\right\rfloor^{\gamma_{u, i}}$

where by adding and subtracting (14) the consensus term (15) can be rewritten as

$$
\Psi_{i}=e_{u, i}+\sum_{j \in \mathcal{N}_{i}} F_{i, j} e_{o, j} .
$$

The choice of $K_{i}$ and $e_{o, j}=\tilde{x}_{o, i}-x_{o, i}=0$ for $i=1, \ldots, N$ leads to

$$
\dot{e}_{u, i}=\mathcal{J}_{r_{i}} e_{u, i}+\operatorname{diag}\left(L_{u, i}\right)\left\lceil 1_{n-r_{i}} e_{u, i, 1}\right\rfloor^{\gamma_{u, i}},
$$

which is homogeneous with degree $\nu_{u, i}$ with respect to the weights $r_{s}=1+(s-1) \nu_{u, i}$. The choice of $L_{u, i}$ and $\nu_{u, i}$ makes the systems 18 globally finite-time stable as shown in Theorem 1 .

Theorem 4 considers the ideal case: When $d(t)=0$ for all $t \geq 0$ (hence the same holds for $\bar{d}_{i}(t)$ ), there is no delay, and the observers for the observable states have

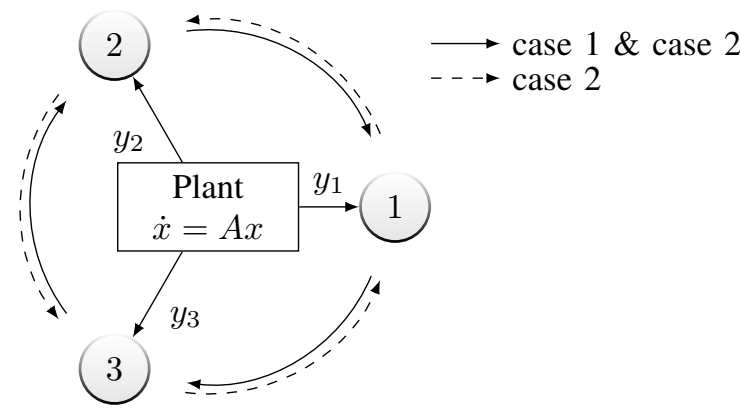

Fig. 1. Distributed observer using 3 observer nodes with 1) undirected and 2) directed communication graph

converged already exactly to their ideal values in a finitetime. By taking into account the robustness properties of homogeneous systems we can now formulate a distributed finite time-observer for the complete state $x$ of the system (4).

Theorem 5: Consider the system (4) with a bounded state $x(t)$ for all $t \geq 0$, an observable pair $\left(A, \operatorname{col}\left\{C_{i}\right\}_{i=1}^{N}\right)$, state transformations for the observability decomposition (5) as well as observers 8 and 16 for the observable and unobservable substates, $i=1, \ldots, N$, respectively. Under Assumption 1 and $\bar{d}_{i}=0$ and $\tau_{i, j}=0$ for all $j \in \mathcal{N}_{i}$ and $i=1, \ldots, N$ the estimates $\tilde{x}_{i}=T_{i}\left[\begin{array}{c}\tilde{x}_{o, i} \\ \tilde{x}_{u, i}\end{array}\right]$ will converge in finite-time to $x$. Moreover, for any bounded disturbance $d(t)$ and delay $\tau_{i, j}$ the error $e_{i}=\tilde{x}_{i}-x$ will remain bounded.

Proof: In the noise and delay-free case, from Theorem 2 follows that there exist a convergence time $t_{1}>0$ for which $e_{o, i}(t) \rightarrow 0$ for $t<t_{1}$ and $\tilde{x}_{o, i}=x_{o, i}$ for $t \geq t_{1}, i=$ $1, \ldots, N$. Then from Theorem 4 directly follows $\tilde{x}_{u, i}=x_{u, i}$ for $t \geq t_{2}>t_{1}$ (the solutions of (16) do not explode on the interval $\left[0, t_{1}\right]$ due to its ISS property, see below).

To complete the proof, it is necessary to consider the boundedness of the trajectories $\tilde{x}_{i}(t)$ in the presence of disturbances, noises and delays. The boundedness of $e_{o, i}(t)$ for $t \geq 0$ follows from the ISS property of homogeneous systems [19]. This in turn guarantees boundedness of $e_{u, i}$, since due to Theorem 4 the system (17) is homogeneous with negative degree for $e_{o, j}=0, j \in \mathcal{N}_{i}$, which implies that in the case of $e_{o, j} \neq 0$ and

$\Psi_{i}(t)=e_{u, j}(t)+\sum_{j \in \mathcal{N}_{i}} F_{i, j}\left(\tilde{x}_{o, i}\left(t-\tau_{i, j}\right)-x_{o, i}(t)\right)-\bar{d}_{i}(t)$

it is also ISS with respect to bounded $e_{o, j}(t)$ (if $\tau_{i, j}=0$ ), bounded $\tilde{x}_{o, i}(t)-F_{i, j} \tilde{x}_{o, j}\left(t-\tau_{i, j}\right)$ (due to boundedness of $x(t)$ ) and bounded $d$. 


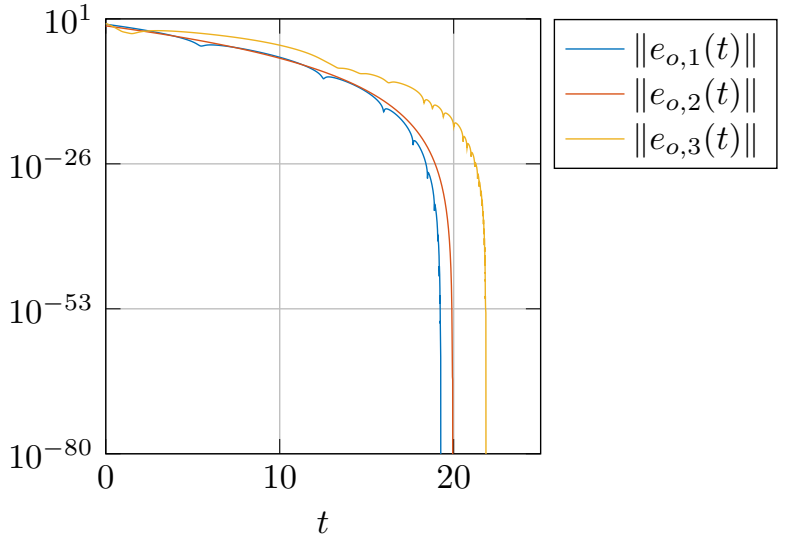

Fig. 2. Error of the observable substate for each observer node in logarithmic scaling

\section{EXAMPLE}

Let us consider an example with $N=3$ observer nodes, see Fig. 1. The plant dynamics is

$$
A=\left[\begin{array}{cccccc}
-1 & 0 & 0 & 0 & 0 & 0 \\
-1 & 1 & 1 & 0 & 0 & 0 \\
1 & -2 & -1 & -1 & 1 & 1 \\
0 & 0 & 0 & 1 & 0 & 0 \\
-8 & 1 & 1 & -1 & -2 & 0 \\
4 & -0 & 0 & 0 & 0 & -4
\end{array}\right]
$$

and the output matrices for each observer node are

$$
\begin{aligned}
C_{1} & =\left[\begin{array}{llllll}
2 & 0 & 0 & 1 & 0 & 0
\end{array}\right], \\
C_{2} & =\left[\begin{array}{llllll}
0 & 0 & 0 & 2 & 0 & 0
\end{array}\right], \\
C_{3} & =\left[\begin{array}{llllll}
2 & 0 & 5 & 0 & 0 & 0
\end{array}\right] ;
\end{aligned}
$$

it is not observable from any single output $\left(r_{1}=2, r_{2}=\right.$ $\left.1, r_{3}=5\right)$.

Each observer node can estimate its observable subspace in finite-time and to this end the gains are chosen as

$$
L_{o, 1}=\left[\begin{array}{l}
2 \\
1
\end{array}\right], L_{o, 2}=1, L_{o, 3}=\left[\begin{array}{c}
5 \\
10 \\
10 \\
5 \\
1
\end{array}\right]
$$

and $\nu_{o, 1}=\nu_{o, 2}=\nu_{o, 3}=-0.05$. Fig. 2 shows a simulation of the error of the observable substate with observers according to Theorem 2. The logarithmic scaling of the $y$-axis make the finite-time conference property clearly visible.

For the remaining substates the observer nodes will have to rely on the other estimates using the consensus term. In the first case the observer nodes are connected in an undirected cyclic graph, which means it is complete and thus Assumption 1 holds. The gains are chosen as in Theorem 4 with

$$
L_{u, 1}=\left[\begin{array}{l}
4 \\
6 \\
4 \\
1
\end{array}\right], L_{u, 2}=\left[\begin{array}{c}
5 \\
10 \\
10 \\
5 \\
1
\end{array}\right], L_{u, 3}=1
$$

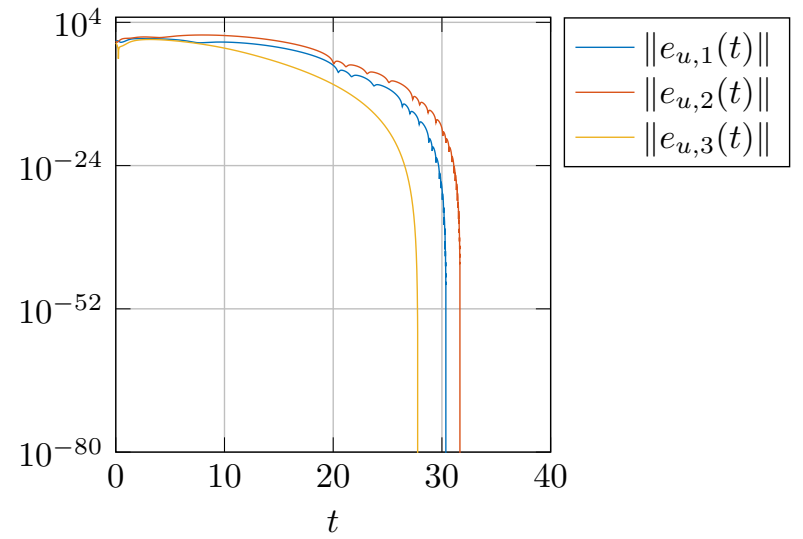

Fig. 3. Error of the unobservable substate for each observer node in logarithmic scaling

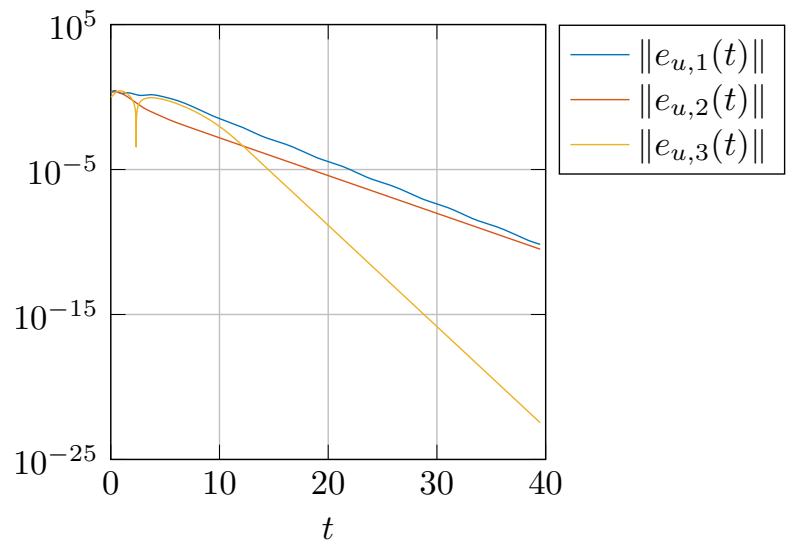

Fig. 4. Error of the unobservable substate for each observer node in logarithmic scaling: asymptotic observer

and $\nu_{u, 1}=\nu_{u, 2}=\nu_{u, 3}=-0.05$. Fig. 3 shows that also the errors in the remaining substates are finite-time stable.

Let us take a look in the case where the graph is cyclic but directed, for example node 1 can send to node 2 , node 2 to node 3 and node 3 to node 1 . In that case Assumption 1 is not fulfilled for the second node (case 2 in Fig. 1). Following Theorem 5 we can still design an asymptotical stable observer. Using YALMIP [22], the gains

$$
\begin{aligned}
K_{1} & =\left[\begin{array}{ccccc}
1.63 & -3.17 & -0.48 & -2.35 \\
-0.74 & 0.58 & 0.25 & 1.09 \\
-0.01 & -1.28 & -3.34 & -0.01 \\
1.48 & 0.84 & -0.39 & -1.58
\end{array}\right] \\
K_{2} & =\left[\begin{array}{ccccc}
-1.38 & -0.06 & -4.01 & -0.02 & 0.20 \\
-0.04 & -0.72 & 8.00 & -0.35 & 1.14 \\
0.00 & 0.00 & -0.40 & 0.00 & -0.00 \\
0.44 & 0.71 & -0.99 & 1.05 & -1.59 \\
-0.19 & -0.40 & 1.00 & -1.28 & 1.83
\end{array}\right] \\
K_{3} & =2.61
\end{aligned}
$$

were obtained for the unobservable part of the observers. Fig. 4 shows the corresponding error when simulated. 


\section{CONClusion}

A distributed finite-time observer design was presented, provided that the plant is observable from the combination of all outputs under a connectivity assumption. When dealing with disturbances, noises and delays, the proposed observer acts as a filter and robustness is guaranteed due to the ISS property of homogeneous systems. For the design the decoupling property of finite-time stability was exploited.

The importance of the connectivity assumption was highlighted by presenting a counterexample with an asymptotically converging observer. Note that in fact it suffices if the assumption is fulfilled by a single node, since if one node is able to estimate its unobservable substate in a finite time, then the neighboring observer nodes can also make use of that estimate. The conditions on the graph for an improvement of this result is a direction of current research.

\section{ACKNOWLEDGMENT}

This work has been partially supported by the project UCoCoS, funded by the European Union's Horizon 2020 research and innovation program under the Marie SkłodowskaCurie Grant Agreement No 675080. It was also partially funded by Russian Science Foundation (Project 17-1901422), the Government of Russian Federation (Grant 074U01) and the Ministry of Education and Science of Russian Federation (Project 14.Z50.31.0031).

\section{REFERENCES}

[1] H. Nijmeijer and I. M. Y. Mareels, "An observer looks at synchronization," IEEE Transactions on Circuits and Systems I: Fundamental Theory and Applications, vol. 44, no. 10, pp. 882-890, Oct. 1997.

[2] L. Wang and A. S. Morse, "A distributed observer for a time-invariant linear system," IEEE Transactions on Automatic Control, vol. 63, no. 7, pp. 2123-2130, Jul. 2018.

[3] S. Park and N. C. Martins, "Design of Distributed LTI Observers for State Omniscience," IEEE Transactions on Automatic Control, vol. 62, no. 2, pp. 561-576, Feb. 2017.

[4] H. Silm, R. Ushirobira, D. Efimov, W. Michiels, J.-P. Richard, and E. Fridman, "Comparison of the timedelay margin of a distributed and centralized observer," in 2018 European Control Conference (ECC), Limassol: European Control Association, Jun. 12, 2018.

[5] T. Kim, H. Shim, and D. D. Cho, "Distributed Luenberger observer design," in Decision and Control (CDC), 2016 IEEE 55th Conference On, IEEE, 2016, pp. 6928-6933.

[6] W. Han, H. L. Trentelman, Z. Wang, and Y. Shen, "A Simple Approach to Distributed Observer Design for Linear Systems," IEEE Transactions on Automatic Control, vol. 64, no. 1, pp. 329-336, Jan. 2019.
[7] Á. R. del Nozal, P. Millán, L. Orihuela, A. Seuret, and L. Zaccarian, "Distributed estimation based on multihop subspace decomposition," Automatica, vol. 99, pp. 213-220, Jan. 1, 2019.

[8] A. Mitra and S. Sundaram, "Distributed Observers for LTI Systems," IEEE Transactions on Automatic Control, vol. 63, no. 11, pp. 3689-3704, Nov. 2018.

[9] R. Olfati-Saber, "Distributed Kalman filtering for sensor networks," in 2007 46th IEEE Conference on Decision and Control, LA, Dec. 2007, pp. 5492-5498.

[10] V. Ugrinovskii, "Distributed robust filtering with $\mathrm{H} \infty$ consensus of estimates," Automatica, vol. 47, no. 1, pp. 1-13, Jan. 1, 2011.

[11] H. Silm, R. Ushirobira, D. Efimov, J. Richard, and W. Michiels, "A Note on Distributed Finite-Time Observers," IEEE Transactions on Automatic Control, vol. 64, no. 2, pp. 759-766, Feb. 2019.

[12] V. Andrieu, L. Praly, and A. Astolfi, "Homogeneous Approximation, Recursive Observer Design, and Output Feedback," SIAM Journal on Control and Optimization, vol. 47, no. 4, pp. 1814-1850, Jan. 1, 2008.

[13] A. Levant, "Homogeneity approach to high-order sliding mode design," Automatica, vol. 41, no. 5, pp. 823830, May 1, 2005.

[14] W. Perruquetti, T. Floquet, and E. Moulay, "FiniteTime Observers: Application to Secure Communication," IEEE Transactions on Automatic Control, vol. 53, no. 1, pp. 356-360, Feb. 2008.

[15] K. Zimenko, D. Efimov, A. Polyakov, and W. Perruquetti, "A note on delay robustness for homogeneous systems with negative degree," Automatica, 2017.

[16] A. Mitra, J. A. Richards, S. Bagchi, and S. Sundaram, "Finite-Time Distributed State Estimation over TimeVarying Graphs: Exploiting the Age-of-Information," Oct. 14, 2018. [Online]. Available: http: / / arxiv. org/abs/1810.06151

[17] T. Kailath, Linear Systems. Prentice-Hall, 1980.

[18] S. P. Bhat and D. S. Bernstein, "Geometric homogeneity with applications to finite-time stability," Mathematics of Control, Signals and Systems, vol. 17, no. 2, pp. 101-127, Jun. 1, 2005.

[19] E. Bernuau, A. Polyakov, D. Efimov, and W. Perruquetti, "Verification of ISS, iISS and IOSS properties applying weighted homogeneity," Systems \& Control Letters, vol. 62, no. 12, pp. 1159-1167, Dec. 1, 2013.

[20] R. Olfati-Saber and J. S. Shamma, "Consensus Filters for Sensor Networks and Distributed Sensor Fusion," in Proceedings of the 44th IEEE Conference on Decision and Control, Dec. 2005, pp. 6698-6703.

[21] D. Spanos and R. M. Murray, "Distributed Sensor Fusion Using Dynamic Consensus," 2005.

[22] J. Lofberg, "YALMIP : A toolbox for modeling and optimization in MATLAB," in 2004 IEEE International Conference on Robotics and Automation, Taipei, Sep. 2004, pp. 284-289. 